\title{
Developing an Interpretable Machine Learning Model to Predict In-Hospital Mortality in Sepsis Patients: A Retrospective Study of MIMIC-IV
}

\author{
Shuhe Li \\ The First Affiliated Hospital of Sun Yat-Sen University \\ Xiaodong Song \\ The First Affiliated Hospital of Sun Yat-Sen University \\ Ka Yin Lui \\ The First Affiliated Hospital of Sun Yat-Sen University \\ Yanping Zhu \\ The First Affiliated Hospital of Sun Yat-Sen University \\ Jinghong $X u$ \\ The First Affiliated Hospital of Sun Yat-Sen University \\ Zilu Guo \\ William March Rice University \\ Xiaoguang Hu \\ The First Affiliated Hospital of Sun Yat-Sen University \\ Xiangdong Guan \\ The First Affiliated Hospital of Sun Yat-Sen University \\ Changjie Cai ( $\nabla$ caichjie@mail.sysu.edu.cn ) \\ The First Affiliated Hospital of Sun Yat-Sen University
}

\section{Research Article}

Keywords: Sepsis, Mortality, Extreme Gradient Boosting, Prediction, Medical Information Mart for Intensive Care IV

Posted Date: February 14th, 2022

DOI: https://doi.org/10.21203/rs.3.rs-1282305/v1

License: @ (i) This work is licensed under a Creative Commons Attribution 4.0 International License. Read Full License 


\section{Abstract}

Background: Risk stratification plays an essential role in the decision-making of sepsis management, whereas neither a single serum biomarker nor traditional scoring tools can satisfy the need to assess this heterogeneous population comprehensively. The study intended to develop an interpretable machine learning model for predicting in-hospital mortality in critically ill patients with sepsis.

Methods: Adult patients fulfilling the definition of Sepsis-3 were included in the Medical Information Mart for Intensive Care (MIMIC)-IV database. Relevant clinical features were extracted within the first 24 hours in ICU, and missing data were analyzed and imputed. We randomly separated the dataset into train and test sub-cohort by the ratio of 7:3, then an outcome-balanced train dataset was synthesized for model training. Extreme gradient boosting (XGBoost) was employed when feature selection and hyperparameter tuning were performed afterward. The fine-tuned XGBoost model was then compared with stepwise logistic regression (LR) and established severity scores. Eventually, we inspected the interpretability of the new model using XGBoost feature importance and Shapley Additive exPlanations (SHAP) plot.

Results: The final cohort had 24,573 patients, of which 3,785 patients died during hospitalization (15.4\%). Ten iterations of multiple imputations were executed to fill missing data in all 91 incomplete variables. Subsequently, 10,572 patients formed the balanced dataset used for training. The XGBoost model showed greater discrimination than stepwise LR and severity scores such as Simplified Acute Physiology Score (SAPS)-III score (AUC: 0.849, 95\% Cl: 0.8386-0.8599; AUC: 0.618, 95\% Cl: 0.3927-0.8437; AUC: 0.803, 95\% Cl: 0.7898-0.8165 respectively). Based on model interpretation, some decisive factors, including elevated lactate and anion gap level, prolonged partial thromboplastin time, decreased urine volume, were greatly correlated with poor survival outcomes in sepsis.

Conclusions: In the field of predicting the mortality risk of sepsis patients in hospitals, the XGBoost-based model demonstrated superior performance to stepwise LR and other scores. In addition, the model exhibited good interpretability and might provide valuable hints on future directions of clinical practice and research.

\section{Background}

Sepsis is a lethal syndrome characterized by multi-organ dysfunction triggered by an irreversible response to infection [1]. Sepsis represents a leading cause of death in intensive care units (ICU), accounting for $6.7 \%$ of all decedents from 2005 to 2018 in the United States [2]. Since early risk stratification in sepsis patients plays a crucial role in bedside decisionmaking and clinical resources allocation, several novel biomarkers and scoring tools have emerged.

Single serum biomarker such as procalcitonin (PCT), interleukin (IL)-6[3], presepsin[4], or CD64 [5] has shown insufficient sensitivity or specificity in practice because the sepsis population is composed of heterogeneous patients caused by diverse etiologies and presented with distinctive manifestations[6]. On the other hand, traditional scoring systems are facing new challenges. For instance, selecting six representative variables and their cut-off values in the Sequential Organ Failure Assessment (SOFA) score was initially determined on expert consensus (7), resulting in a weak rationale foundation. Additionally, most long-established predictive scores like Acute Physiology and Chronic Health Evaluation (APACHE) score are based on binary logistic regression (LR) equations [7], which have a strict requirement on no intercorrelation and limited ability dealing with missingvalues [8], even though multicollinearity and missingness are both frequently observed in real-world data. Nevertheless, in the era of electronic health data explosion and sophisticated machine learning $(\mathrm{ML})$ technique advancement, employing data-driven $\mathrm{ML}$ models into ICU daily practice has signaled a promising expansion on the possibility of healthcare [9]. Researchers have already developed ML-based severity scores in critically ill patients with postoperative sepsis[10], influenza[11], cerebral hemorrhage [12], and so on. Despite that, the 'black-box' nature of ML had remained a significant obstacle for clinicians to fully trust the advice generated from computational algorithms[9]. 
Hence, in the present study, we intended to develop an interpretable machine learning model to estimate in-hospital mortality in sepsis patients, utilizing highly-dimensional real-world data from the latest Medical Information Mart for Intensive Care (MIMIC)-IV database. A robust supervised algorithm named extreme gradient boosting (XGBoost) was implemented, capable of processing multicollinearity and missing data with high efficiency. It was also compared with the conventional LR model and other severity scores. Moreover, we employed visualized interpretation on the XGBoost-based model to provide novel insights on routinely collected clinical features.

\section{Methods}

Database and subject selection

The study utilized an open-source critical care database MIMIC-IV (Version 1.0) $[13,14]$. The database enrolled over three hundred thousand patients admitted at Beth Israel Deaconess Medical Center (BIDMC) from 2008 to 2019. MIMIC-IV contained highly granular clinical data, including hourly-recorded vital signs received from bedside monitors, laboratory measurements, infusion medications, intake and output volumes, etc. To gain access to MIMIC-IV, one author (S Li) had completed the Collaborative Institutional Training Initiative (CITI) training program 'Human Research, Data or Specimens Only Research' (Record ID: 32396061).

Cohort selection was executed according to the third international consensus definition, Sepsis-3 [1], where sepsis was screened satisfying both suspected host infection (determined by ordering a microbiology culture or antibiotics) and a SOFA score $\geq 2$. Patients who were under 18 years old, had repetitive ICU admissions except for the first time, or stayed in ICU less than 24 hours were all excluded.

Variable and outcome

We performed variable extraction with PostgreSQL (Version 14.0; PostgreSQL Global Development Group). Features were selected within 24 hours after ICU admission, involving the following domains: (1) Demographic characteristics, including age, gender, ethnicity, admitted ICU type, comorbidities, etc. (2) Vital signs, for example, heart rate, respiratory rate, systolic/diastolic/mean blood pressure (BP). Maximum, minimum, and mean values were all abstracted. (3) Laboratory measurements, e.g., blood routines, liver and kidney functions, coagulations, arterial blood gas indexes. The highest and lowest values were extracted. (4) Clinical intervention, comprising of invasive ventilation (i.e., tracheal intubation or tracheostomy), non-invasive oxygen therapy (including standard oxygen delivery, high-flow nasal cannula (HFNC), and non-invasive ventilation (NIV)), renal replacement therapy (RRT), vasopressor usage and maximal doses (including dobutamine, dopamine, epinephrine, norepinephrine, phenylephrine, and vasopressin), time to culture sampling and antibiotic administration. Urine output, source of infection, and Glasgow Coma Scales (GCS) were also included. Furthermore, Simplified Acute Physiology Score (SAPS)-II, SAPS-III, Logistic Organ Dysfunction Score (LODS), Oxford Acute Severity of IIIness Score (OASIS), and SOFA score acted as benchmarks for evaluating the performance of the new model. The outcome was the probability of in-hospital death of every sepsis patient.

Statistical analysis and missing data management

All statistical analysis and machine learning tasks were conducted on R (Version 3.6.3; R Foundation for Statistical Computing), frequently wielded packages included 'xgboost', 'mlr', 'mice', 'DMwR', etc. Univariate analysis was performed between survivors and non-survivors. Continuous variables were described as mean and standard deviation (SD) or median and interquartile range (IQR), compared by the Student's t-test or one-way analysis of variance (ANOVA) test as appropriate. Categorical variables were presented as number and percentage and were compared by Pearson's chi-square test. A two-sided $\mathrm{P}<0.05$ was considered statistically significant. 
Since varied extents of absence were observed in the raw dataset, we visualized missing patterns on every feature and patient, respectively. Features with absent proportions above $50 \%$ were excluded from the ML model. Missing records were imputed based on predictive mean matching. In this robust multiple imputation method, missing values of nonrespondents were randomly attributed by observed values from respondents with the closest predicted means[15]. The absence of maximal vasopressor doses was regarded as non-usage; thus, those would not be imputed. Additionally, the imputation bias was appraised by scatter graphs of the imputed and original data points from features with over $25 \%$ missingness.

Machine learning model development and interpretation

Initially, we separated the final cohort into train and test sub-cohorts through reproducible random sampling without replacement by the ratio of 7:3. Because of the imbalance of outcome classifications, where non-survivors constituted only $15.4 \%$ of all patients, the autonomous learning process from non-survivors could be poorly influenced. Therefore, a balanced train dataset (train-SMOTE) was synthetically generated from the original train cohort through oversampling the minority event [16], i.e., in-hospital death, fulfilled by the 'SMOTE' function in package 'DMwR'. Besides, we preserved the test cohort without imputation to examine model performance in a real-world setting.

Next, we employed XGBoost to predict the probability of in-hospital mortality with eligible variables in the train-SMOTE dataset. XGBoost is a decision-tree-based algorithm first proposed by Chen et al. [17]. Weak learners are ensembled iteratively into a strong predictor; in the meantime, the loss function is minimized through gradient boosting. The initial parameters were set as follows: learning object as 'binary: logistic', evaluation metrics as 'logloss', learning rate (eta) as 0.05 , iterations (nround) as 5,000, and early stopping rounds as 25 . Then feature selection was performed using the cumulative feature importance score obtained from the default model until all included variables ascended to $90 \%$. Lastly, to optimize the ML model, we executed hyperparameter tuning through five-fold cross-validation (CV), i.e., 4 out of 5 equivalent sub-samples were used for training and the remaining one for validation. Optimal values were obtained through 20 rounds of CV iterations in the previously defined ranges (Table S1). The final parameters fed into ML model were listed as followed: learning rate (eta $=0.1274431)$, maximum depth of a tree (max_depth $=8$ ), minimum loss reduction to make a further leaf node (gamma $=1.354569)$, subsample proportion (subsample $=0.8297109)$, subsample ratio of columns when constructing a tree (colsample_by_tree $=0.6685429)$, minimum sum of instance weight needed in a child node (min_child_weight $=3$ ), iterations (nround $=102)$, L2 regularization (lambda $=28.0494)$, and randomized seed number (seed.number $=731$ ).

On top of that, we also developed a multivariate logistic regression model using the same features as the XGBoost model. A stepwise selection of variables was conducted in both forward and backward directions, where Akaike Information Criterion (AIC) was used for determining the inclusion or exclusion of a particular feature into the LR model. Besides, severity score-based models such as SOFA and SAPS were built after training in the train-SMOTE cohort.

Subsequently, the discrimination and interpretability of new models were investigated. We used receiver operating characteristic (ROC) curves and area under the curve (AUC), sensitivity, specificity, accuracy to compare the discriminatory ability of LR and XGBoost models in train and test datasets, respectively. The performance of XGBoost was also compared with the mentioned severity scores in the test cohort. Furthermore, feature importance and Shapley Additive exPlanations (SHAP) summary plot were implemented to illustrate included features' contributions and potential impacts on in-hospital mortality risk.

\section{Results}

Patient characteristics 
Figure 1 presented a total of 24,573 patients fulfilling the inclusion criteria in MIMIC-IV, of whom $15.4 \%$ died in hospital (N $=3,785)$. 17,177 patients were randomly assigned to the train cohort and 7,396 patients were designated to the test cohort. Demographic characteristics and clinical features between survivors and non-survivors of all patients were shown in Table 1 and Table S2. The median age of our cohort was $66 \pm 16$ years, and $57.9 \%$ were male patients. Potential bloodborne infection was detected in more than half of all sepsis patients. Non-survivors tended to be admitted through emergency department (ED) and treated in coronary care unit (CCU) and medical intensive care unit (MICU) (all P<0.001), supported by Medicare insurance $(P<0.001)$, more likely to have comorbidities such as cerebrovascular disorders (CVD) and cancers (all $P<0.001)$, urinate less $(1009[475,1796]$ vs. $1668[1065,2480] \mathrm{mL}, \mathrm{P}<0.001)$, and acquire higher SOFA scores in contrast to survivors $(4.361 \pm 2.518$ vs. $3.499 \pm 1.871, \mathrm{P}<0.001)$. Non-survivors presented significantly worse vital signs and laboratory values, for instance, elevated maximum white blood cell (WBC) count $(15.0$ [10.2, 20.8] vs. 13.7 $\left.[9.9,18.4] \times 10^{9} / \mathrm{L}, \mathrm{P}<0.001\right)$, increased maximum serum lactate and anion gap (AG) $(3.0[1.7,5.8]$ vs. $2.2[1.5,3.4]$ $\mathrm{mmol} / \mathrm{L}, \mathrm{P}<0.001 ; 18[15,22]$ vs. $15[13,18] \mathrm{mmol} / \mathrm{L}, \mathrm{P}<0.001$ respectively), worse liver and kidney functions and prolonged coagulation indexes (all $\mathrm{P}<0.001)$, decreased minimum oxygenation index $(143.33[86.13,237.25]$ vs. 192.00 $[123.00,275.00] \mathrm{mmHg}, \mathrm{P}<0.001)$, etc. Besides, non-survivors were inclined to receive invasive ventilation $(48.7 \%$ vs. $40.9 \%, \mathrm{P}<0.001)$, whereas non-invasive oxygen therapy was applied in survival patients more frequently $(54.2 \% \mathrm{vs}$. $37.5 \%, \mathrm{P}<0.001)$. Non-survivors also had a higher possibility administering RRT and vasopressors (all $\mathrm{P}<0.001$ ), especially norepinephrine (43.2\% vs. $21.6 \%, \mathrm{P}<0.001)$. Lastly, non-survivors experienced a more delayed course of initiating antibiotics $(6.218 \pm 6.314$ vs. $5.483 \pm 5.994$ hours following ICU admission, $P<0.001)$, taking cultures $(0.422 \pm$ 7.839 vs. $0.972 \pm 7.889$ hours before ICU admission, $P=0.698$ ), and a more rapid onset of sepsis attack than survivors (1 $[0,4]$ vs. $3[1,6]$ hours following ICU admission, $P<0.001)$.

Missingness and imputation

Figure S1 demonstrated the pattern of missingness in the original dataset according to each feature and patient separately. Among all 149 features, 14 features had missing proportions above $50 \%$ and were excluded from model development. Some laboratory features shared similar missing patterns, such as alanine aminotransferase (ALT), aspartate aminotransferase (AST), alkaline phosphatase (ALP), and total bilirubin, for the reason that they were likely to be ordered as a bundle by physicians since they reflected the function of a specific organ. In total, ten iterations of multiple imputations were executed in 91 incomplete variables. Figure 2 visualized the imputation process of features with missing percentages above $25 \%$, indicating that the distribution of imputed values matched the existing ones. Additionally, a synthetic dataset (train-SMOTE) was generated to improve the learning process from both survivors and non-survivors ( $N=10,572)$, where the in-hospital mortality rate was lifted to $50 \%$ (Table S3).

\section{Model performance}

After stepwise variable selection, 92 features were included in the logistic regression model. As demonstrated in Table 2, the following features were correlated with increased in-hospital death risks: advanced age (OR: 1.025, 95\% Cl: 1.0201.030), admitted by ED (OR: 1.392, 95\% Cl: 1.144-1.693), pre-existing CVD or cancer (OR: 2.314, 95\% Cl: 1.980-2.704; OR: 2.404, 95\% Cl: 2.055-2.811 respectively), potential bloodstream infection (OR: 1.211, 95\% Cl: 1.066-1.377), invasive mechanical ventilation (OR: 1.667, 95\% Cl: 1.452-1.914), norepinephrine usage (OR: 1.297, 95\% Cl: 1.125-1.495), and so on. Contrarily, surgical admission (OR: $0.440,95 \% \mathrm{Cl}$ : 0.310-0.626), non-invasive oxygen therapy (OR: 0.536, 95\% Cl: 0.471-0.610), and elevated minimum GCS (OR: 0.969, 95\% Cl: 0.950-0.987) were linked to greater survival benefits. Interestingly, every hour delayed initiating antimicrobial therapy or obtaining culture was likely to increase mortality risk by 1.3\% and 3.6\%, respectively (OR: 1.013, 95\% Cl: 1.003-1.022; OR: 1.036, 95\% Cl: 1.026-1.046). Discrimination of LR model during training was excellent (AUC: $0.911,95 \% \mathrm{Cl}: 0.9059-0.9164)$, whereas performance was unsatisfactory in the test cohort due to incapacity of processing missingness (AUC: $0.618,95 \% \mathrm{Cl}: 0.3927-0.8437$ ) (Table 3 ). 
The final XGBoost model was trained with optimal fine-tuned hyperparameters listed in the former section. As Figure 3 showed, the train and test dataset log-loss values during cross-validation were 0.168666 and 0.379745 separately at the best iterations of 102 rounds, indicating an acceptable degree of overfitting. XGBoost model exhibited appealing discriminatory ability in both train and test dataset (AUC: $0.995,95 \%$ Cl: 0.9943-0.9959; AUC: 0.849, 95\% Cl: 0.8386-0.8599 respectively) (Table 3). Furthermore, XGBoost model outperformed well-acknowledged scores for predicting hospitalization outcomes of sepsis patients, including SAPS-III (AUC: 0.803, 95\% Cl: 0.7898-0.8165), LODS (AUC: 0.782, 95\% Cl: 0.7671-0.7959), OASIS (AUC: 0.739, 95\% Cl: 0.7242-0.7547), SAPS-II (AUC: 0.737, 95\% Cl: 0.7216-0.7524), and SOFA score (AUC: $0.598,95 \% \mathrm{Cl}$ : 0.5799-0.6161). (Figure 4)

Model interpretability

The XGBoost model was visually interpreted to provide compelling insights into the clinical domain. Figure 5 demonstrated feature importance rankings in XGBoost, implying that estimating in-hospital mortality was heavily dependent on clinical features such as maximum AG, minimum lactate, maximum partial thromboplastin time (PTT), urine output of the first day, etc. (Gain: $0.116,0.080,0.056,0.035$ respectively) On top of that, SHAP summary plot revealed that risk factors including higher lactate, PTT, AG and red cell distribution width (RDW) levels, elderly and an underlying CVD, faster respiratory rate and lower systolic BP were all associated with poor survival outcomes (Figure 6). However, increased urine production and non-invasive oxygen therapy might have a protective effect on sepsis patients.

\section{Discussion}

This study created an interpretable machine learning model for in-hospital mortality prediction in ICU patients diagnosed with sepsis. The XGBoost model showed superior discrimination to the conventional LR model and other established scores, including SAPS-III and SOFA scores. We also explored essential clinical factors and their specific impacts on inhospital death among sepsis patients, such as AG, lactate, PTT, urine production, and non-invasive oxygen therapy. The current study could propose novel clues for further understanding of sepsis.

Most acknowledged severity scores are created from multivariate logistic regression equations [7, 18, 19]. Traditional LR models are well interpretable and easy to understand, though they bear limitations such as a necessity for satisfying linearity relationship, rejecting multicollinearity [8], unable to handle missing data, etc. Considering the complexity of the sepsis population, more sophisticated and mathematical algorithms were expected. Nowadays, more and more predictive models have been developed using machine learning techniques, targeting various aspects of the sepsis population, including diagnosis, prognosis, and management. For instance, Kong et al. [20] developed several ML-based models to predict the hospital mortality of sepsis patients based on the MIMIC-III database, utilizing demographic features and clinical variables such as acute physiological measurements in the first 24 hours in ICU and chronic health status. The study suggested that the gradient boosting machine (GBM) model demonstrated the highest AUC (0.845) and good calibration compared to other ML models and SAPS-II. On top of that, a deep learning-based autonomous pipeline has been recently constructed, capable of offering hourly-refreshed probabilities on sepsis patients' survival [21]. This powerful tool incorporated a broad scope of structured or unstructured data in MIMIC-III, alleviating workload from manual variable selection and data curation. The model showed good discrimination at 48 hours (AUC: 0.8463 ) and could guide ICU stepdown strategies among low-risk patients.

Machine learning has promising prospects in augmenting disease pattern identification and therapeutic strategy individualization, because of its capacity to efficiently process multi-dimensional and multi-variety big data and continuously improve performance from new data. In this study, the XGBoost model outperformed logistic regression and other long-established scoring tools after being trained with the same dataset, showing AUCs as high as $0.995,0.849$ in the train and test cohort separately. Nevertheless, machine learning has not been so widely acknowledged and applied in the field of critical care as that in marketing, autonomous vehicles, social media, and other domains. Despite the emerging 
numbers of new publications, few examples of data-driven decision-supporting systems have been successfully run in hospital settings. One major issue is that clinicians could not have trusted the advice from these complicated ML-based models, if they could not understand the rationale behind them, which contrarily lied the strength of logistic regression. Hence, good interpretability has become a necessity in model development, not only for researchers to verify the reliability of new predictive models, but also for clinicians to gain confidence in sharing decision-making responsibilities with computational algorithms [9]. From our interpretable ML model, we have discovered several compelling predictors that were crucially related to outcomes in sepsis patients, which might hint at future directions of clinical practice and research.

First, serum lactate and AG were presented as the most critical factors contributing to sepsis mortality prediction. The higher lactate and AG levels were, the greater morality risks were based on the SHAP plot. Data had shown that critically ill patients with metabolic acidosis were about twice as likely to die as patients without acidosis (45\% vs. 25\%) [22], when lactic and strong ion acidosis were two primary sources. Serum lactate has been suggested as a decisive risk factor to sepsis patients, as Liu et al. [23] found that discrimination of lactate was superior to quick-SOFA score and similar to SOFA score (AUC: $0.664,0.547,0.686$, respectively). Besides, an elevated AG is reported to be associated with higher rates of ICU admission[24], increased severity of disease, and in-hospital mortality compared to patients with average AG values[25]. In a retrospective cohort of 4,159 sepsis patients admitted to $E D$, an $A G \geq 20 \mathrm{mEq} / \mathrm{L}$ displayed a similar likelihood ratio in predicting mortality compared to lactate $>2[26]$. Since Survival Sepsis Campaign's (SSC) 1-hour bundle [27] has been broadly promoted, serum lactate has become a routine measurement in ICU settings; therefore, introducing lactate or AG into novel scoring systems should be advocated.

Secondly, non-invasive oxygen therapy, including standard oxygen delivery, HFNC, and NIV, might be protective on sepsis patients (OR: 0.536, $\mathrm{P}=0.001$ ), whereas implementing invasive mechanical ventilation indicated a poor survival as the LR model illustrated (OR: 1.667, P < 0.001). Interpreted from the XGBoost model, non-invasive oxygen therapy was also one of the most important predictors for sepsis mortality (Gain: 0.013), leading to improved outcomes. Oxygen supplementation has been commonly applied in patients with sepsis, when few trials nor recommendations are focused explicitly on different oxygenation strategies on sepsis cohort[28]. A multicenter randomized trial has reported that HFNC was correlated with increased ventilator-free days and reduced 90-day mortality compared with oxygen therapy and NIV in patients with acute hypoxemic respiratory failure[29]. However, studies targeting HFNC on sepsis were either terminated early such as OPTISEPSIS (NCT03334227), or not yet recruiting (NCT04560842).

In addition, decreased urine volume and prolonged PTT represented impaired renal and coagulation functions separately; thus, they were among the top five pivotal risk factors as XGBoost feature importance and SHAP plot revealed. Lastly, according to odds ratios from the stepwise LR model, postponed time to administer antibiotics was related to significantly higher death risks (OR:1.013, $\mathrm{P}=0.012)$, so was obtaining culture (OR:1.036, $\mathrm{P}<0.001)$. Likewise, Seymour and his colleagues[30] investigated the completion status of a 3-hour bundle on sepsis patients treated in EDs in 149 hospitals.

They found that more extended time to initiate antimicrobial therapy or obtain a blood culture were both associated with a $4 \%$ increase in in-hospital mortality risk per hour (OR:1.04, 95\% Cl: 1.03-1.06; OR:1.04, 95\% Cl: 1.02-1.06 respectively). Hence, more prompt recognition and infection control was a decisive key to survival among sepsis patients.

Compared to previous publications, our study showed several strengths. Firstly, we employed the latest MIMIC-IV database to develop our models, as it included critically ill patients in a long but contemporary period from 2008 to 2019. In addition, every three years as an interval was evaluated for risk stratification to avoid healthcare disparities resulting from dynamic clinical guidance and recommendations. Second, the XGBoost model was significantly more efficient in learning high-order interactions and non-linear functions than conventional logistic regression. Furthermore, good interpretability was intently assessed in our ML model to provide promising clues on future practice and research. Despite that, the current study also had the following limitations. This study was a single centered retrospective analysis; therefore, potential bias was inevitable. Moreover, clinical variables were considered only within the first 24 hours in the

Page $7 / 24$ 
ICU, like the APACHE-II score, so progressive physiological status or therapeutic intervention was not fully incorporated into the current model. Third, the model had not been validated in external datasets other than the independent test dataset in our cohort; therefore, further validation was anticipated to examine its generalizability.

\section{Conclusion}

In conclusion, this study suggested that an XGBoost-based model outperformed conventional logistic regression and severity scores such as SOFA and SAPS-III in predicting in-hospital mortality among sepsis patients in the ICU environment. Additionally, this model partially alleviated the 'black-box' issue and demonstrated good interpretability utilizing XGBoost feature importance and SHAP summary plot, indicating lactate, AG, PTT, urine output, non-invasive oxygen therapy, and other features as vital predictors linked to survival outcomes. Future evidence is warranted regarding validating the mentioned predictors and continuously developing predictive models with advanced ML approaches.

\section{Declarations}

\section{Ethics approval and consent to participate}

Research use of MIMIC-IV data has been approved by the Institutional ReviewBoard of the BIDMC and Massachusetts Institute of Technology. The author had completed the Collaborative Institutional Training Initiative (CITI) training program 'Human Research, Data or Specimens Only Research' to gain access to the database, therefore requirement for individual patient consent was waived because of the deidentified natureof MIMIC-IV. All methods were executed under relevant guidelines and regulations.

\section{Consent for publication}

Not applicable.

\section{Availability of data and material}

The dataset supporting the conclusions of this article is MIMIC-IV, available at https://mimic.physionet.org.

\section{Competing interests}

None to declare.

\section{Funding}

No funding.

\section{Authors' contributions}

SL participated in the conception and design, statistical analysis, data interpretation, drafting, and manuscript revisions. $X S, K L, Y Z, J X, X H$ participated in study conception and design, data interpretation, and manuscript modifications. ZG participated in revisions of statistical analysis, model development, and data interpretation. XG and CC participated in study conception and design, manuscript revisions, and study progress supervision. All authors read and approved the manuscript for publication.

\section{Acknowledgments}

We appreciate the marvelous work of the Laboratory for Computational Physiology (Massachusetts Institute of Technology) to collect bedside data consistently and share them with intensive care researchers over the world non- 
profitably.

\section{References}

1. Singer M, Deutschman CS, Seymour CW, et al. The Third International Consensus Definitions for Sepsis and Septic Shock (Sepsis-3). JAMA 2016, 315:801-810.

2. Prest J, Sathananthan M, Jeganathan N. Current Trends in Sepsis-Related Mortality in the United States. Crit Care Med 2021, 49:1276-1284.

3. Henning DJ, Hall MK, Watsjold BK, et al. Interleukin-6 improves infection identification when added to physician judgment during evaluation of potentially septic patients. The American Journal of Emergency Medicine 2020, 38:947-952.

4. Nakamura Y, Hoshino K, Kiyomi F, et al. Comparison of accuracy of presepsin and procalcitonin concentrations in diagnosing sepsis in patients with and without acute kidney injury. Clin Chim Acta 2019, 490:200-206.

5. Dimoula A, Pradier O, Kassengera Z, et al. Serial determinations of neutrophil CD64 expression for the diagnosis and monitoring of sepsis in critically ill patients. Clin Infect Dis 2014, 58:820-829.

6. Pierrakos C, Velissaris D, Bisdorff M, et al. Biomarkers of sepsis: time for a reappraisal. Critical Care 2020, 24:287.

7. Zimmerman JE, Kramer AA, McNair DS, et al. Acute Physiology and Chronic Health Evaluation (APACHE) IV: hospital mortality assessment for today's critically ill patients. Crit Care Med 2006, 34:1297-1310.

8. Midi H, Sarkar SK, Rana S. Collinearity diagnostics of binary logistic regression model. Journal of Interdisciplinary Mathematics 2010, 13:253-267.

9. Sanchez-Pinto LN, Luo Y, Churpek MM. Big Data and Data Science in Critical Care. Chest 2018, 154:1239-1248.

10. Yao RQ, Jin X, Wang GW, et al. A Machine Learning-Based Prediction of Hospital Mortality in Patients With Postoperative Sepsis. Front Med (Lausanne) 2020, 7:445.

11. Hu CA, Chen CM, Fang YC, et al. Using a machine learning approach to predict mortality in critically ill influenza patients: a cross-sectional retrospective multicentre study in Taiwan. BMJ Open 2020, 10:e033898.

12. Nie X, Cai Y, Liu J, et al. Mortality Prediction in Cerebral Hemorrhage Patients Using Machine Learning Algorithms in Intensive Care Units. Front Neurol 2020, 11:610531.

13. Johnson A, Bulgarelli L, Pollard T, et al. 'MIMIC-IV' (version 1.0). PhysioNet 2021.

14. Goldberger A, Amaral L, Glass L, et al. PhysioBank, PhysioToolkit, and PhysioNet: Components of a new research resource for complex physiologic signals. Circulation [Online] 2000, 101:e215-e220.

15. Morris TP, White IR, Royston P. Tuning multiple imputation by predictive mean matching and local residual draws. BMC Medical Research Methodology 2014, 14:75.

16. Chawla NV, Bowyer KW, Hall LO, et al. SMOTE: Synthetic Minority Over-sampling Technique. Journal of Artificial Intelligence Research 2002, 16:321-357.

17. Chen T, Guestrin C. XGBoost: A Scalable Tree Boosting System. 2016.

18. Le Gall JR, Klar J, Lemeshow S, et al. The Logistic Organ Dysfunction system. A new way to assess organ dysfunction in the intensive care unit. ICU Scoring Group. Jama 1996, 276:802-810.

19. Moreno RP, Metnitz PGH, Almeida E, et al. SAPS 3-From evaluation of the patient to evaluation of the intensive care unit. Part 2: Development of a prognostic model for hospital mortality at ICU admission. Intensive Care Medicine 2005, 31:1345-1355.

20. Kong G, Lin K, Hu Y. Using machine learning methods to predict in-hospital mortality of sepsis patients in the ICU. BMC Med Inform Decis Mak 2020, 20:251. 
21. Deasy J, Lio P, Ercole A. Dynamic survival prediction in intensive care units from heterogeneous time series without the need for variable selection or curation. Sci Rep 2020, 10:22129.

22. Gunnerson KJ, Saul M, He S, et al. Lactate versus non-lactate metabolic acidosis: a retrospective outcome evaluation of critically ill patients. Critical Care 2006, 10:R22.

23. Liu Z, Meng Z, Li Y, et al. Prognostic accuracy of the serum lactate level, the SOFA score and the qSOFA score for mortality among adults with Sepsis. Scandinavian journal of trauma, resuscitation and emergency medicine 2019 , 27:51-51.

24. Brenner BE. Clinical significance of the elevated anion gap. Am J Med 1985, 79:289-296.

25. Sahu A, Cooper HA, Panza JA. The initial anion gap is a predictor of mortality in acute myocardial infarction. Coron Artery Dis 2006, 17:409-412.

26. Mohr NM, Vakkalanka JP, Faine BA, et al. Serum anion gap predicts lactate poorly, but may be used to identify sepsis patients at risk for death: a cohort study. 2018, 44:223-228.

27. Levy MM, Evans LE, Rhodes AJIcm. The surviving sepsis campaign bundle: 2018 update. 2018, 44:925-928.

28. Perner A, De Jong A, Shankar-Hari M. Trials on oxygen supplementation in sepsis: better late than never. Intensive Care Medicine 2020, 46:116-118.

29. Frat J-P, Thille AW, Mercat A, et al. High-Flow Oxygen through Nasal Cannula in Acute Hypoxemic Respiratory Failure. New England Journal of Medicine 2015, 372:2185-2196.

30. Seymour CW, Gesten F, Prescott HC, et al. Time to Treatment and Mortality during Mandated Emergency Care for Sepsis. N Engl J Med 2017, 376:2235-2244.

\section{Tables}

Table 1. Baseline features and clinical interventions of 24,573 sepsis patients categorized by in-hospital mortality. 


\begin{tabular}{|c|c|c|c|c|}
\hline Features & $\begin{array}{l}\text { Total } \\
(\mathrm{N}=24,573)\end{array}$ & $\begin{array}{l}\text { Survivor } \\
(\mathrm{N}=20,788)\end{array}$ & $\begin{array}{l}\text { Non- } \\
\text { survivor(N=3,785) }\end{array}$ & $\begin{array}{l}\mathrm{P}- \\
\text { value }\end{array}$ \\
\hline Male, \% & $14222(57.9 \%)$ & $12147(58.4 \%)$ & $2075(54.8 \%)$ & $<0.001$ \\
\hline Age, years & $66.041(16.184)$ & 65.371(16.244) & 69.720(15.338) & $<0.001$ \\
\hline \multicolumn{5}{|l|}{ Ethnicity, \% } \\
\hline Caucasian & $16561(67.4 \%)$ & $14255(68.6 \%)$ & $2306(60.9 \%)$ & $<0.001$ \\
\hline African American & $2099(8.5 \%)$ & $1801(8.7 \%)$ & $298(7.9 \%)$ & 0.117 \\
\hline Hispanic & $829(3.4 \%)$ & $724(3.5 \%)$ & $105(2.8 \%)$ & 0.030 \\
\hline Asian & $697(2.8 \%)$ & $593(2.9 \%)$ & $104(2.7 \%)$ & 0.761 \\
\hline Other/Unknown & 4387(17.9\%) & $3415(16.4 \%)$ & $972(25.7 \%)$ & $<0.001$ \\
\hline Height,cm & $169.561(10.625)$ & 169.783(10.613) & 168.397(10.613) & $<0.001$ \\
\hline Weight,kg & $79.0[66.1,94.2]$ & $79.6[66.8,94.8]$ & $76.0[63.5,91.1]$ & $<0.001$ \\
\hline \multicolumn{5}{|l|}{ AdmittedICU,\% } \\
\hline CVICU & $5329(21.7 \%)$ & $5101(24.5 \%)$ & $228(6.0 \%)$ & $<0.001$ \\
\hline $\mathrm{CCU}$ & $2222(9.0 \%)$ & $1731(8.3 \%)$ & $491(13.0 \%)$ & $<0.001$ \\
\hline MICU & $5377(21.9 \%)$ & $4297(20.7 \%)$ & $1080(28.5 \%)$ & $<0.001$ \\
\hline SICU & $3397(13.8 \%)$ & $2865(13.8 \%)$ & $532(14.1 \%)$ & 0.673 \\
\hline MICU/SICU & $4535(18.5 \%)$ & $3638(17.5 \%)$ & 897(23.7\%) & $<0.001$ \\
\hline TSICU & $2811(11.4 \%)$ & $2400(11.5 \%)$ & $411(10.9 \%)$ & 0.233 \\
\hline Neuro-ICU & $902(3.7 \%)$ & $756(3.6 \%)$ & $146(3.9 \%)$ & 0.537 \\
\hline \multicolumn{5}{|l|}{ Admissiontype,\% } \\
\hline ObservationAdmit & $2332(9.5 \%)$ & 1912(9.2\%) & $420(11.1 \%)$ & $<0.001$ \\
\hline SurgicalAdmit & $2611(10.6 \%)$ & $2535(12.2 \%)$ & $76(2.0 \%)$ & $<0.001$ \\
\hline Elective & $1157(4.7 \%)$ & $1113(5.4 \%)$ & $44(1.2 \%)$ & $<0.001$ \\
\hline Emergency & $13314(54.2 \%)$ & 10989(52.9\%) & $2325(61.4 \%)$ & $<0.001$ \\
\hline Urgent & $5159(21.0 \%)$ & $4239(20.4 \%)$ & $920(24.3 \%)$ & $<0.001$ \\
\hline \multicolumn{5}{|l|}{ Insurancetype,\% } \\
\hline Medicaid & $1742(7.1 \%)$ & $1486(7.1 \%)$ & $256(6.8 \%)$ & 0.416 \\
\hline Medicare & $11433(46.5 \%)$ & $9434(45.4 \%)$ & 1999(52.8\%) & $<0.001$ \\
\hline Other & $11398(46.4 \%)$ & $9868(47.5 \%)$ & $1530(40.4 \%)$ & $<0.001$ \\
\hline \multicolumn{5}{|l|}{ Maritalstatus, $\%$} \\
\hline Single & $6389(26.0 \%)$ & $5569(26.8 \%)$ & $820(21.7 \%)$ & $<0.001$ \\
\hline Married & $11113(45.2 \%)$ & $9580(46.1 \%)$ & $1533(40.5 \%)$ & $<0.001$ \\
\hline Divorced/Widowed & $4865(19.8 \%)$ & $4071(19.6 \%)$ & $794(21.0 \%)$ & 0.050 \\
\hline
\end{tabular}


Admitted period, \%

\begin{tabular}{|c|c|c|c|c|}
\hline $2008-2010$ & $8483(34.5 \%)$ & $7291(35.1 \%)$ & 1192(31.5\%) & $<0.001$ \\
\hline $2011-2013$ & $6119(24.9 \%)$ & $5242(25.2 \%)$ & $877(23.2 \%)$ & 0.008 \\
\hline 2014-2016 & $5885(23.9 \%)$ & $4967(23.9 \%)$ & $918(24.3 \%)$ & 0.648 \\
\hline 2017-2019 & $4086(16.6 \%)$ & $3288(15.8 \%)$ & $798(21.1 \%)$ & $<0.001$ \\
\hline \multicolumn{5}{|l|}{ Comorbidities } \\
\hline $\mathrm{MI}, \%$ & $4365(17.8 \%)$ & $3561(17.1 \%)$ & $804(21.2 \%)$ & $<0.001$ \\
\hline $\mathrm{CHF}, \%$ & 7378(30.0\%) & $6006(28.9 \%)$ & $1372(36.2 \%)$ & $<0.001$ \\
\hline CVD, \% & $3640(14.8 \%)$ & $2849(13.7 \%)$ & $791(20.9 \%)$ & $<0.001$ \\
\hline CPD, \% & $6495(26.4 \%)$ & $5421(26.1 \%)$ & $1074(28.4 \%)$ & 0.003 \\
\hline CKD, \% & $5370(21.9 \%)$ & $4342(20.9 \%)$ & $1028(27.2 \%)$ & $<0.001$ \\
\hline Diabetes mellitus, \% & $7507(30.5 \%)$ & $6366(30.6 \%)$ & $1141(30.1 \%)$ & 0.557 \\
\hline Cancer, \% & $3826(15.6 \%)$ & 2855(13.7\%) & $971(25.7 \%)$ & $<0.001$ \\
\hline \multicolumn{5}{|l|}{ Source of infection } \\
\hline Bloodstream, \% & $13560(55.2 \%)$ & $10899(52.4 \%)$ & $2661(70.3 \%)$ & $<0.001$ \\
\hline Respiratory tract, \% & $4126(16.8 \%)$ & $3173(15.3 \%)$ & $953(25.2 \%)$ & $<0.001$ \\
\hline Cerebrospinal fluid, \% & $373(1.5 \%)$ & $317(1.5 \%)$ & $56(1.5 \%)$ & 0.834 \\
\hline GI/Peritoneal, \% & $2249(9.2 \%)$ & $1737(8.4 \%)$ & $512(13.5 \%)$ & $<0.001$ \\
\hline Genitourinary, \% & $7125(29.0 \%)$ & $5967(28.7 \%)$ & $1158(30.6 \%)$ & 0.018 \\
\hline \multicolumn{5}{|l|}{ Severity of disease } \\
\hline SOFA & $3.632(2.009)$ & $3.499(1.871)$ & $4.361(2.518)$ & $<0.001$ \\
\hline LODS & $5.836(3.377)$ & $5.274(3.015)$ & 8.923(3.581) & $<0.001$ \\
\hline SAPS-II & $39.810(14.160)$ & $37.813(12.941)$ & $50.775(15.490)$ & $<0.001$ \\
\hline SAPS-III & $55.474(25.536)$ & $50.859(21.996)$ & $80.821(28.578)$ & $<0.001$ \\
\hline OASIS & $34.944(9.220)$ & $33.672(8.679)$ & $41.924(8.978)$ & $<0.001$ \\
\hline $\begin{array}{l}\text { Time to antibiotics administration, } \\
\text { hour }\end{array}$ & $5.596(6.050)$ & $5.483(5.994)$ & $6.218(6.314)$ & $<0.001$ \\
\hline Time to culture sampling, hour* & $0.887(7.883)$ & $0.972(7.889)$ & $0.422(7.839)$ & 0.698 \\
\hline $\begin{array}{l}\text { Time to sepsis onset, } \\
\text { hour }\end{array}$ & $2[1,6]$ & $3[1,6]$ & $1[0,4]$ & $<0.001$ \\
\hline Urine output, $\mathrm{mL}$ & $1580[962,2400]$ & $1668[1065,2480]$ & $1009[475,1795.5]$ & $<0.001$ \\
\hline Minimum GCS & $15[13,15]$ & $15[13,15]$ & $15[12,15]$ & $<0.001$ \\
\hline $\begin{array}{l}\text { Invasive ventilation, } \\
\%\end{array}$ & $10354(42.1 \%)$ & $8511(40.9 \%)$ & $1843(48.7 \%)$ & $<0.001$ \\
\hline Non-invasive oxygen therapy, & $12696(51.7 \%)$ & $11275(54.2 \%)$ & $1421(37.5 \%)$ & $<0.001$ \\
\hline
\end{tabular}




\begin{tabular}{|c|c|c|c|c|}
\hline RRT, \% & $773(3.1 \%)$ & $538(2.6 \%)$ & $235(6.2 \%)$ & $<0.001$ \\
\hline \multicolumn{5}{|l|}{ Vasopressor } \\
\hline $\begin{array}{l}\text { Dobutamine usage, } \\
\%\end{array}$ & $340(1.4 \%)$ & $209(1.0 \%)$ & $131(3.5 \%)$ & $<0.001$ \\
\hline $\begin{array}{l}\text { Maximum dobutamine dose, } \\
\mathrm{mcg} / \mathrm{kg} / \mathrm{min}\end{array}$ & $5.002[2.503,7.012]$ & $5.002[2.502,5.055]$ & $5.005[2.504,7.510]$ & 0.13 \\
\hline $\begin{array}{l}\text { Dopamine usage, } \\
\%\end{array}$ & $753(3.1 \%)$ & $514(2.5 \%)$ & $239(6.3 \%)$ & $<0.001$ \\
\hline $\begin{array}{l}\text { Maximum dopamine dose, } \\
\mathrm{mcg} / \mathrm{kg} / \mathrm{min}\end{array}$ & $18.373(152.436)$ & 19.848(182.190) & $15.203(43.117)$ & $<0.001$ \\
\hline $\begin{array}{l}\text { Epinephrine usage, } \\
\%\end{array}$ & $1477(6.0 \%)$ & $1185(5.7 \%)$ & $292(7.7 \%)$ & $<0.001$ \\
\hline $\begin{array}{l}\text { Maximum epinephrine dose, } \\
\mathrm{mcg} / \mathrm{kg} / \mathrm{min}\end{array}$ & $0.130(1.102)$ & 0.064(0.199) & $0.397(2.430)$ & $<0.001$ \\
\hline $\begin{array}{l}\text { Norepinephrine usage, } \\
\%\end{array}$ & $6127(24.9 \%)$ & $4493(21.6 \%)$ & $1634(43.2 \%)$ & $<0.001$ \\
\hline $\begin{array}{l}\text { Maximum norepinephrine dose, } \\
\mathrm{mcg} / \mathrm{kg} / \mathrm{min}\end{array}$ & $0.180[0.100,0.331]$ & $0.150[0.090,0.300]$ & $0.300[0.143,0.496]$ & $<0.001$ \\
\hline $\begin{array}{l}\text { Phenylephrine usage, } \\
\%\end{array}$ & $6164(25.1 \%)$ & $5317(25.6 \%)$ & $847(22.4 \%)$ & $<0.001$ \\
\hline $\begin{array}{l}\text { Maximum phenylephrine dose, } \\
\mathrm{mcg} / \mathrm{kg} / \mathrm{min}\end{array}$ & $1.001[0.600,2.002]$ & $1.001[0.500,1.988]$ & $2.196[1.200,4.074]$ & $<0.001$ \\
\hline $\begin{array}{l}\text { Vasopressin usage, } \\
\%\end{array}$ & $1639(6.7 \%)$ & $968(4.7 \%)$ & 671(17.7\%) & $<0.001$ \\
\hline $\begin{array}{l}\text { Maximum vasopressin dose, } \\
\text { units/min }\end{array}$ & $3.150(13.007)$ & $3.040(9.903)$ & $3.309(16.493)$ & 0.084 \\
\hline
\end{tabular}

CVICU, Cardiovascular Intensive Care Unit; CCU, Coronary Care Unit; MICU, Medical Intensive Care Unit; SICU, Surgical Intensive Care Unit; TSICU, Trauma Surgical Intensive Care Unit;MI, Myocardial infarction; CHF, Congestive Heart Failure; CVD, Cerebrovascular Disorder; CPD, Chronic Pulmonary Disease; CKD, Chronic Kidney Disease; GI, Gastrointestinal; SOFA, Sequential Organ Failure Assessment; LODS, Logistic Organ Dysfunction Score; SAPS, Simplified Acute Physiology Score; OASIS, Oxford Acute Severity of Illness Score; GCS, Glasgow Coma Scale; RRT, Renal Replacement Therapy.

*Only time to culture sampling represented time periods before ICU admission.

Table 2. Multivariate stepwise logistic regression model for in-hospital mortalityamong sepsis patients. 


\begin{tabular}{|c|c|c|c|c|}
\hline Features & OR & $2.5 \% \mathrm{Cl}$ & $97.5 \% \mathrm{Cl}$ & P-value \\
\hline Male(female as reference) & 0.763 & 0.653 & 0.890 & 0.001 \\
\hline Admission age (per year) & 1.025 & 1.020 & 1.030 & $<0.001$ \\
\hline \multicolumn{5}{|c|}{ Ethnicity(Other/Unknown as reference) } \\
\hline Asian & 1.459 & 1.026 & 2.073 & 0.035 \\
\hline African & 0.464 & 0.359 & 0.602 & $<0.001$ \\
\hline Hispanic & 1.104 & 0.805 & 1.514 & 0.539 \\
\hline Caucasian & 0.742 & 0.624 & 0.881 & 0.001 \\
\hline Height (per cm) & 1.015 & 1.007 & 1.023 & $<0.001$ \\
\hline Weight (per kg) & 0.992 & 0.989 & 0.995 & $<0.001$ \\
\hline \multicolumn{5}{|c|}{ Admitted ICU(CVICU as reference) } \\
\hline $\mathrm{CCU}$ & 1.515 & 1.162 & 1.975 & 0.002 \\
\hline MICU & 1.163 & 0.922 & 1.467 & 0.203 \\
\hline MICU/SICU & 0.890 & 0.695 & 1.138 & 0.353 \\
\hline Neuro-ICU & 1.069 & 0.742 & 1.539 & 0.722 \\
\hline SICU & 0.923 & 0.721 & 1.181 & 0.524 \\
\hline TSICU & 1.097 & 0.846 & 1.423 & 0.486 \\
\hline \multicolumn{5}{|c|}{ Admission type(Observation admit as reference) } \\
\hline Emergency & 1.392 & 1.144 & 1.693 & 0.001 \\
\hline Elective & 0.469 & 0.284 & 0.776 & 0.003 \\
\hline Surgical Admit & 0.440 & 0.310 & 0.626 & $<0.001$ \\
\hline Urgent & 1.201 & 0.962 & 1.499 & 0.105 \\
\hline \multicolumn{5}{|c|}{ Marital status(Other as reference) } \\
\hline Divorced/Widowed & 0.488 & 0.385 & 0.619 & $<0.001$ \\
\hline Married & 0.486 & 0.391 & 0.605 & $<0.001$ \\
\hline Single & 0.454 & 0.360 & 0.572 & $<0.001$ \\
\hline \multicolumn{5}{|c|}{ Admitted period(2008-2010 as reference) } \\
\hline 2011-2013 & 0.979 & 0.840 & 1.141 & 0.786 \\
\hline 2014-2016 & 0.842 & 0.716 & 0.990 & 0.038 \\
\hline 2017-2019 & 0.745 & 0.616 & 0.901 & 0.002 \\
\hline CVD & 2.314 & 1.980 & 2.704 & $<0.001$ \\
\hline CPD & 0.858 & 0.752 & 0.979 & 0.023 \\
\hline Cancer & 2.404 & 2.055 & 2.811 & $<0.001$ \\
\hline Bloodstream & 1.211 & 1.066 & 1.377 & 0.003 \\
\hline
\end{tabular}




\begin{tabular}{|c|c|c|c|c|}
\hline Respiratory tract & 1.576 & 1.366 & 1.819 & $<0.001$ \\
\hline CSF & 0.721 & 0.459 & 1.134 & 0.157 \\
\hline GI/Peritoneal & 1.503 & 1.268 & 1.781 & $<0.001$ \\
\hline Urine output (per mL) & 1.000 & 1.000 & 1.000 & $<0.001$ \\
\hline Minimum GCS & 0.969 & 0.950 & 0.987 & 0.001 \\
\hline Invasive ventilation & 1.667 & 1.452 & 1.914 & $<0.001$ \\
\hline Non-invasive oxygen therapy & 0.536 & 0.471 & 0.610 & $<0.001$ \\
\hline Dopamine usage & 2.318 & 1.642 & 3.272 & $<0.001$ \\
\hline Norepinephrine usage & 1.297 & 1.125 & 1.495 & $<0.001$ \\
\hline Phenylephrine usage & 1.185 & 1.009 & 1.391 & 0.038 \\
\hline Vasopressin usage & 3.013 & 1.997 & 4.545 & $<0.001$ \\
\hline Maximum vasopressin dose (per units/min) & 0.713 & 0.606 & 0.839 & $<0.001$ \\
\hline Maximum norepinephrine dose (per mcg/kg/min) & 0.928 & 0.842 & 1.023 & 0.132 \\
\hline Maximum dopamine dose (per mcg/kg/min) & 0.951 & 0.927 & 0.975 & $<0.001$ \\
\hline Time to antibiotics administration (per hour) & 1.013 & 1.003 & 1.022 & 0.012 \\
\hline Time to culture sampling (per hour) & 1.036 & 1.026 & 1.046 & $<0.001$ \\
\hline Time to sepsis onset (per hour) & 0.967 & 0.953 & 0.981 & $<0.001$ \\
\hline Maximum heart rate (per bpm) & 1.006 & 1.001 & 1.010 & 0.017 \\
\hline Mean heart rate (per bpm) & 1.006 & 1.000 & 1.013 & 0.057 \\
\hline Maximum systolic BP (per mmHg) & 1.004 & 1.000 & 1.007 & 0.027 \\
\hline Minimum diastolic BP (per mmHg) & 0.993 & 0.984 & 1.001 & 0.103 \\
\hline Maximum diastolic BP (per mmHg) & 0.991 & 0.986 & 0.996 & $<0.001$ \\
\hline Mean diastolic BP (per $\mathrm{mmHg}$ ) & 1.027 & 1.009 & 1.045 & 0.003 \\
\hline Maximum mean BP (per mmHg) & 1.004 & 1.001 & 1.007 & 0.005 \\
\hline Mean mean BP (per mmHg) & 0.971 & 0.955 & 0.987 & 0.001 \\
\hline Maximum respiratory rate (per bpm) & 0.974 & 0.962 & 0.986 & $<0.001$ \\
\hline Mean respiratory rate (per bpm) & 1.097 & 1.075 & 1.120 & $<0.001$ \\
\hline Maximum temperature $\left(\right.$ per $\left.^{\circ} \mathrm{C}\right)$ & 1.317 & 1.156 & 1.501 & $<0.001$ \\
\hline Mean temperature $\left(\right.$ per $\left.^{\circ} \mathrm{C}\right)$ & 0.573 & 0.481 & 0.683 & $<0.001$ \\
\hline Minimum SpO2 & 0.985 & 0.973 & 0.997 & 0.012 \\
\hline Maximum SpO2 & 1.086 & 1.008 & 1.170 & 0.030 \\
\hline Mean SpO2 & 0.918 & 0.876 & 0.963 & $<0.001$ \\
\hline \multirow[t]{2}{*}{ Minimum WBC (per $\left.\times 10^{9} / \mathrm{L}\right)$} & 1.048 & 1.031 & 1.066 & $<0.001$ \\
\hline & 0.974 & 0.963 & 0.986 & $<0.001$ \\
\hline
\end{tabular}

Page 15/24 
Maximum WBC (per $\left.\times 10^{9} / \mathrm{L}\right)$

\begin{tabular}{|c|c|c|c|c|}
\hline Minimum hematocrit & 1.082 & 1.015 & 1.153 & 0.016 \\
\hline Minimum hemoglobin (per g/dL) & 0.775 & 0.641 & 0.937 & 0.009 \\
\hline Maximum platelets (per $\times 10^{9} / \mathrm{L}$ ) & 0.999 & 0.999 & 1.000 & 0.012 \\
\hline Minimum MCH (per pg) & 0.910 & 0.838 & 0.988 & 0.025 \\
\hline Maximum MCHC (per g/dL) & 1.095 & 1.009 & 1.188 & 0.029 \\
\hline Maximum MCV (per fl) & 1.050 & 1.022 & 1.078 & $<0.001$ \\
\hline Minimum RBC (per × $\left.10^{12} / \mathrm{L}\right)$ & 1.430 & 1.121 & 1.823 & 0.004 \\
\hline Maximum RBC (per $\left.\times 10^{12} / \mathrm{L}\right)$ & 0.728 & 0.600 & 0.883 & 0.001 \\
\hline Minimum RDW & 0.937 & 0.868 & 1.011 & 0.092 \\
\hline Maximum RDW & 1.158 & 1.080 & 1.241 & $<0.001$ \\
\hline Minimum BUN (per mg/dL) & 1.025 & 1.015 & 1.034 & $<0.001$ \\
\hline Maximum BUN (per mg/dL) & 0.987 & 0.980 & 0.995 & 0.002 \\
\hline Minimum creatinine (per mg/dL) & 0.906 & 0.852 & 0.964 & 0.002 \\
\hline Minimum bicarbonate (per mmol/L) & 0.964 & 0.936 & 0.993 & 0.016 \\
\hline Maximum bicarbonate (per mmol/L) & 0.930 & 0.902 & 0.958 & $<0.001$ \\
\hline Maximum sodium (per mmol/L) & 1.087 & 1.065 & 1.110 & $<0.001$ \\
\hline Minimum potassium (per mmol/L) & 1.163 & 1.042 & 1.297 & 0.007 \\
\hline Minimum chloride (per mmol/L) & 0.919 & 0.901 & 0.937 & $<0.001$ \\
\hline Maximum calcium (per mg/dL) & 1.084 & 1.017 & 1.156 & 0.013 \\
\hline Maximum AG (per mmol/L) & 0.980 & 0.958 & 1.003 & 0.083 \\
\hline Maximum ALT (per IU/L) & 1.000 & 1.000 & 1.000 & 0.020 \\
\hline Minimum ALP (per IU/L) & 1.002 & 1.001 & 1.004 & 0.013 \\
\hline Maximum ALP (per IU/L) & 0.998 & 0.996 & 1.000 & 0.021 \\
\hline Minimum AST (per IU/L) & 1.000 & 1.000 & 1.000 & 0.030 \\
\hline Maximum AST (per IU/L) & 1.000 & 1.000 & 1.000 & 0.004 \\
\hline Minimum total bilirubin (per mg/dL) & 1.031 & 1.017 & 1.045 & $<0.001$ \\
\hline Minimum glucose (per mmol/L) & 1.001 & 1.000 & 1.003 & 0.096 \\
\hline Maximum glucose (per mmol/L) & 0.999 & 0.999 & 1.000 & 0.027 \\
\hline Minimum PT (per s) & 0.965 & 0.921 & 1.010 & 0.122 \\
\hline Maximum PT (per s) & 1.039 & 1.012 & 1.067 & 0.005 \\
\hline Maximum PTT (per s) & 1.006 & 1.004 & 1.008 & $<0.001$ \\
\hline Minimum INR & 1.443 & 0.920 & 2.264 & 0.110 \\
\hline
\end{tabular}




\begin{tabular}{lllll} 
Maximum INR & 0.745 & 0.579 & 0.958 & 0.022 \\
\hline Minimum lactate $($ per mmol/L) & 1.202 & 1.125 & 1.285 & $<0.001$ \\
\hline Maximum lactate $($ per mmol/L) & 1.065 & 1.021 & 1.112 & 0.003 \\
\hline Maximum pH & 3.382 & 0.775 & 14.764 & 0.105 \\
\hline Maximum pO2 (per mmHg) & 0.998 & 0.998 & 0.999 & $<0.001$ \\
\hline Minimum pCO2 (per mmHg) & 0.982 & 0.973 & 0.992 & $<0.001$ \\
\hline Maximum pCO2 (per mmHg) & 0.995 & 0.988 & 1.001 & 0.110 \\
\hline Minimum AADo2 (per mmHg) & 1.002 & 1.002 & 1.003 & $<0.001$ \\
\hline Minimum OI (per mmHg) & 0.999 & 0.999 & 1.000 & 0.023 \\
\hline Maximum OI (per mmHg) & 1.003 & 1.002 & 1.003 & $<0.001$ \\
\hline Minimum BE (per mmol/L) & 1.025 & 1.003 & 1.047 & 0.023 \\
\hline Maximum BE (per mmol/L) & 0.859 & 0.817 & 0.903 & $<0.001$ \\
\hline Maximum TCO2 (per mmol/L) & 1.172 & 1.126 & 1.220 & $<0.001$
\end{tabular}

OR, odds ratio; Cl, confidence interval;CVICU, Cardiovascular Intensive Care Unit; CCU, Coronary Care Unit; MICU, Medical Intensive Care Unit; SICU, Surgical Intensive Care Unit; TSICU, Trauma Surgical Intensive Care Unit; CVD, Cerebrovascular Disorder; CPD, Chronic Pulmonary Disease; CSF, cerebrospinal fluid; GI, Gastrointestinal; GCS, Glasgow Coma Scale; BP, blood pressure; SpO2, peripheral oxygen saturation; WBC, white blood cell; $\mathrm{MCH}$, mean corpuscular hemoglobin; $\mathrm{MCHC}$, mean corpuscular hemoglobin concentration; MCV, mean corpuscular volume; RBC, red blood cell; RDW, red blood cell distribution width; BUN, blood urea nitrogen; AG, anion gap; ALT, alanine transaminase; ALP, alkaline phosphatase; AST, aspartate transaminase; PT, prothrombin time; PTT, partial thromboplastin time; INR, international normalized ratio; pO2, partial pressure of oxygen; pCO2, partial pressure of carbon dioxide; AADO2, alveolar-arterial oxygen gradient; OI, oxygenation index; $\mathrm{BE}$, base excess; TCO2, total carbon dioxide.

Table 3. Discrimination of logistic regressionand XGBoost models in train-SMOTE and test sub-cohorts.

\begin{tabular}{lllll}
\multirow{2}{*}{ Model } & \multicolumn{2}{l}{ Train-SMOTE(N=10,572) } & Test(N=7,396) \\
\cline { 2 - 5 } & LR & XGBoost & LR & XGBoost \\
\hline AUC & 0.911 & 0.995 & 0.618 & 0.8493 \\
\hline $95 \% \mathrm{Cl}$ & $0.9059-0.9164$ & $0.9943-0.9959$ & $0.3927-0.8437$ & $0.8386-0.8599$ \\
\hline Cut-off point & 0.505 & 0.455 & 0.947 & 0.276 \\
\hline Sensitivity & $80.31 \%$ & $96.95 \%$ & $53.33 \%$ & $83.45 \%$ \\
\hline Specificity & $84.66 \%$ & $96.37 \%$ & $81.82 \%$ & $70.99 \%$ \\
\hline Accuracy & $82.48 \%$ & $96.66 \%$ & $65.38 \%$ & $72.92 \%$
\end{tabular}

LR, logistic regression; XGBoost, extreme gradient boosting; SMOTE, synthetic minority over-sampling technique; AUC, area under the curve; $\mathrm{Cl}$, confidence interval.

\section{Figures}




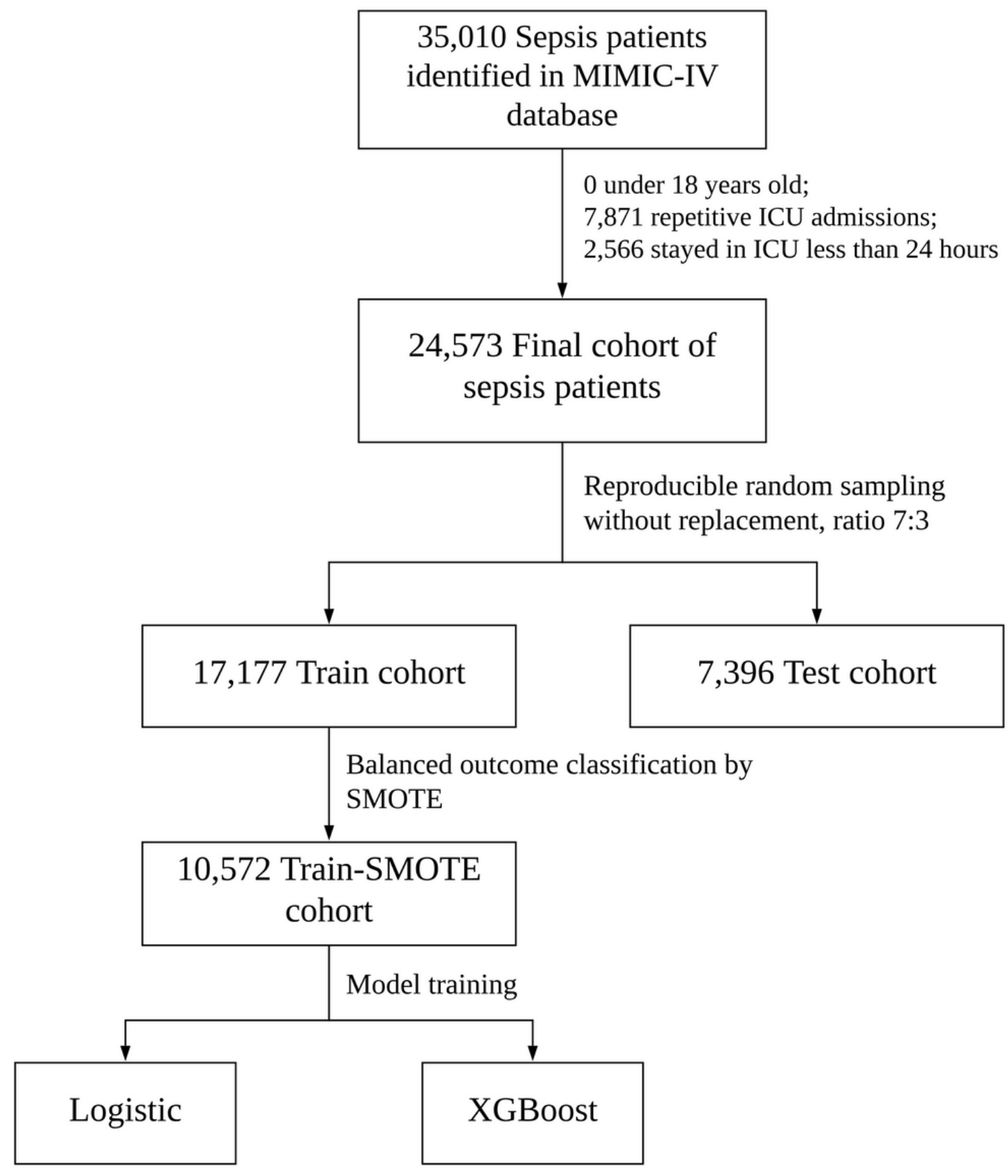

Figure 1

Flowchart of cohort selection. 

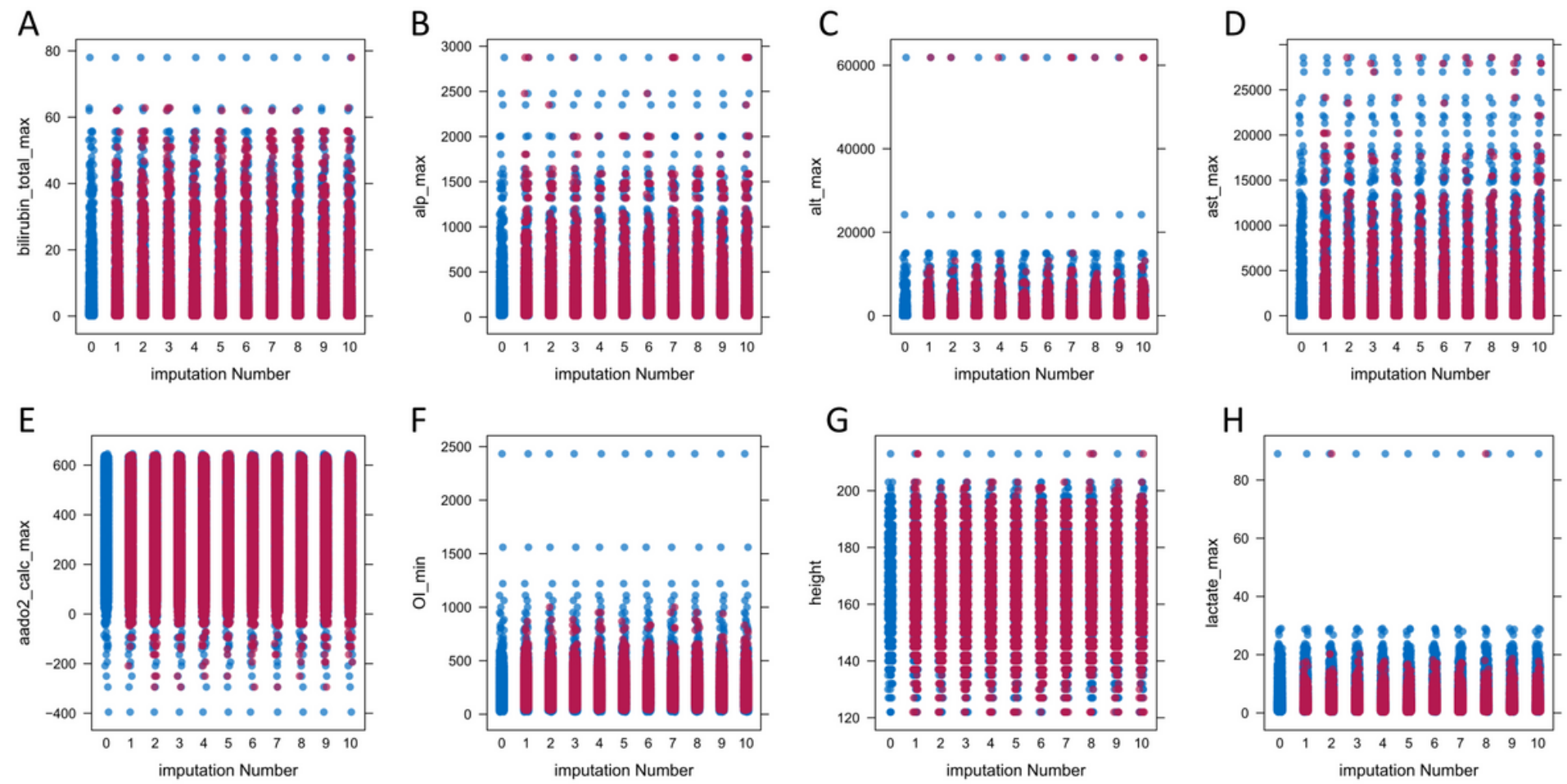

Figure 2

The distribution of original and imputed data points from features with over $25 \%$ missingness. A: Maximum total bilirubin; B: Maximum ALP; C: Maximum ALT; D: Maximum AST; E: Maximum AADO2; F: Minimum Ol; G: Height; H: Maximum lactate.

Blue spots represented pre-existing data and red spots represented imputed data.ALP, alkaline phosphatase; ALT, alanine transaminase; $\mathrm{AST}$, aspartate transaminase; AAD 02, alveolar-arterial oxygen gradient; OI, oxygenation index. 


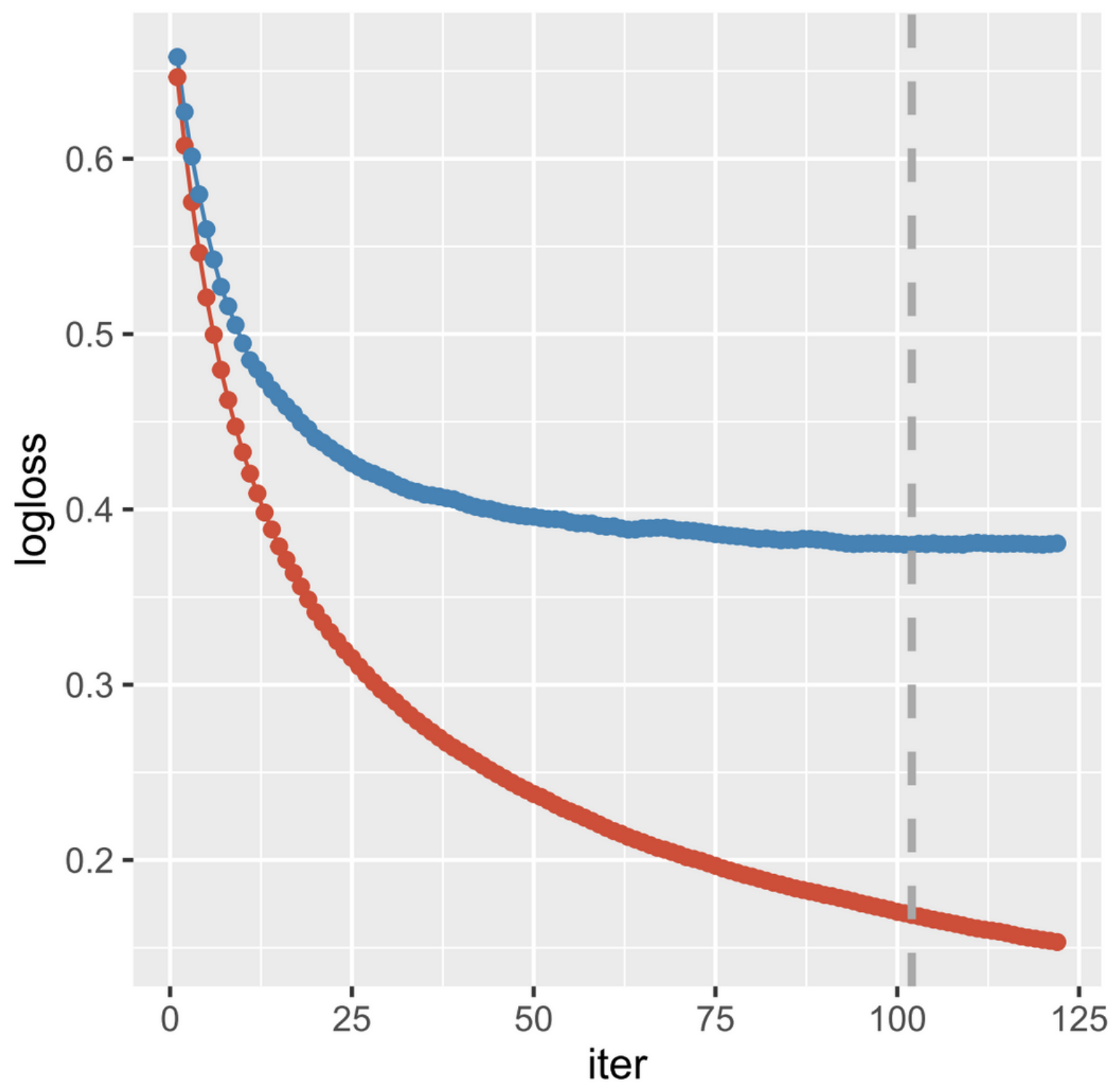

group $\rightarrow$ CV test $\rightarrow$ CV train

Figure 3

The trend of evaluation metric (log-loss values) of XGBoost model together with iterations in the process of cross validation.

Blue lines represented the test cohort and red lines represented the train cohort.XGBoost, extreme gradient boosting. 


\section{ROC Curve}

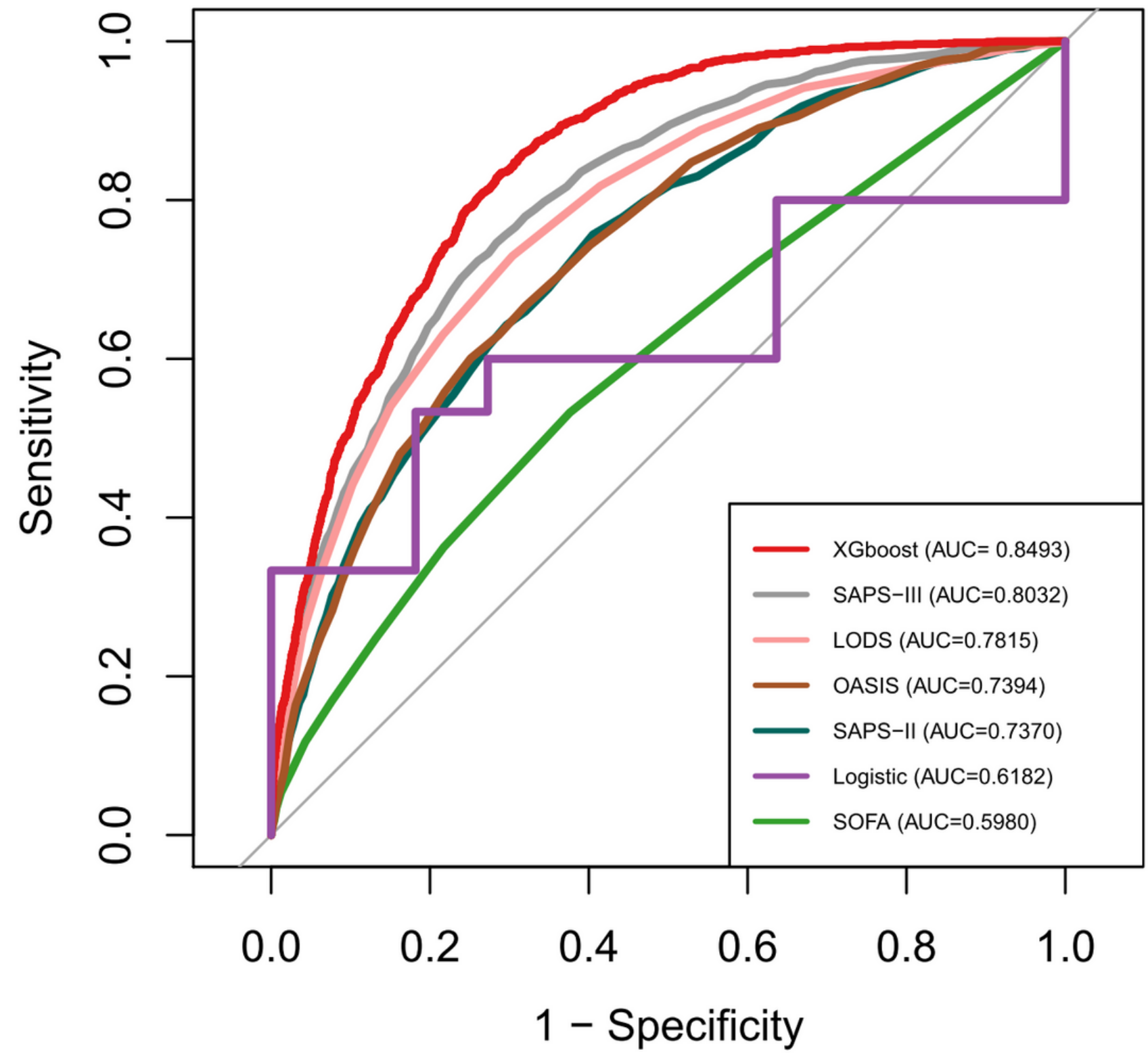

Figure 4

ROC curves presenting the performance of XGBoost model in contrast with LR and established severity scores for estimating in-hospital mortality in the test cohort.

ROC, receiver operating characteristics; XGBoost, extreme gradient boosting; LR, logistic regression; SAPS, simplified acute physiology score; LODS, logistic organ dysfunction score; OASIS, oxford acute severity of illness score; SOFA, sequential organ failure assessment. 


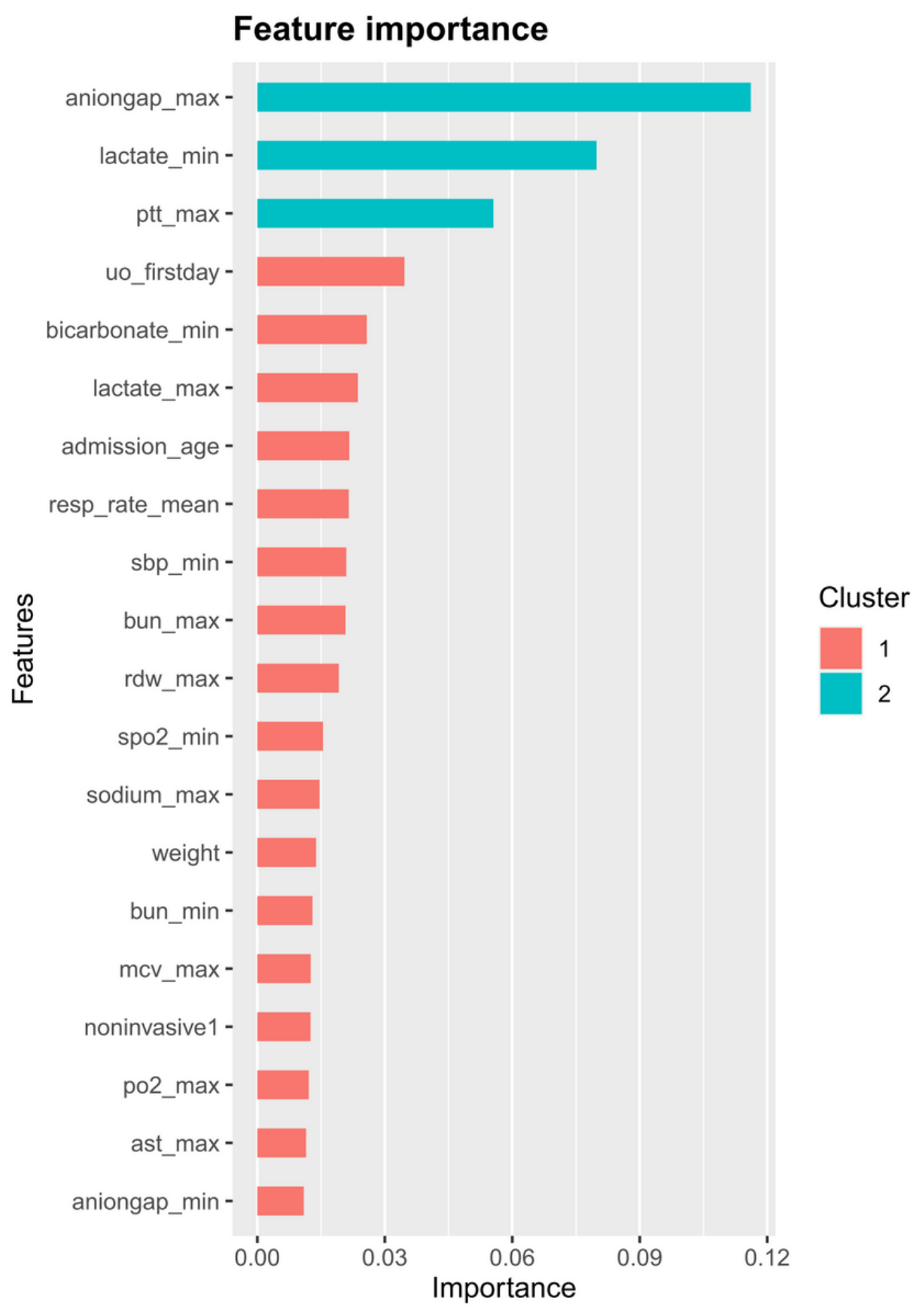

Figure 5

Feature importance rankings inXGBoost model (top 20 features included).

XGBoost, extreme gradient boosting; PTT, partial thromboplastin time;UO, urine output; Resp rate, respiratory rate; SBP, systolic blood pressure; BUN, blood urea nitrogen; RDW, red blood cell distribution width; SpO2, peripheral oxygen saturation; MCV, mean corpuscular volume; noninvasive, non-invasive oxygen therapy; p02, partial pressure of oxygen; AST, aspartate transaminase. 


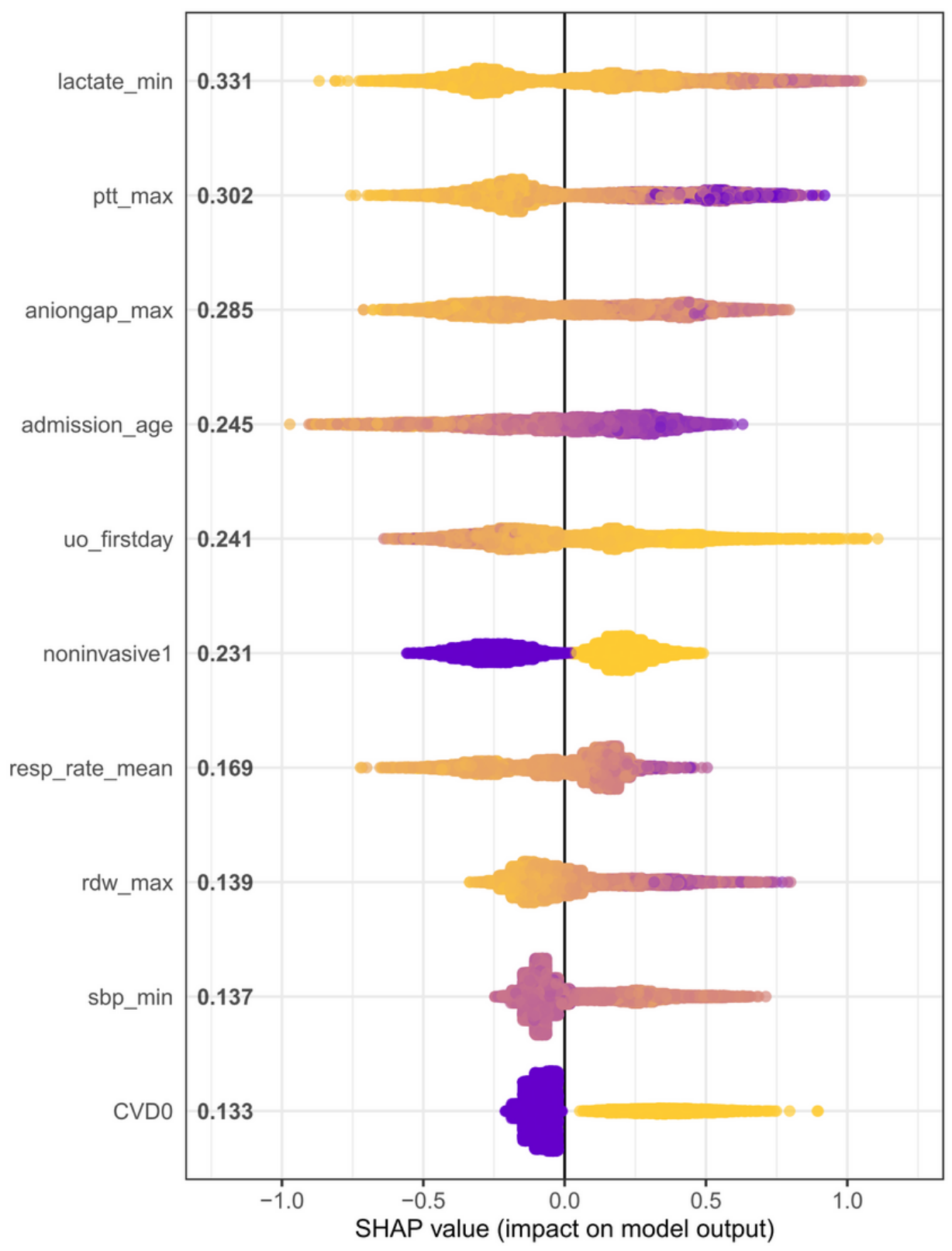

Feature value ${ }_{\text {Low }}$

High

Figure 6

The SHAP summary plot of XGBoost model for predicting in-hospital mortality of sepsis (top 10 features included).

SHAP, Shapley Additive exPlanations; XGBoost, extreme gradient boosting; PTT, partial thromboplastin time; UO, urine output; noninvasive, non-invasive oxygen therapy; Resp rate, respiratory rate;RDW, red blood cell distribution width;SBP, systolic blood pressure; CVD, cerebrovascular disorder.

\section{Supplementary Files}

This is a list of supplementary files associated with this preprint. Click to download.

- SupplementaryMaterials.docx 
- FigureS1.tif

Page 24/24 